\title{
Terahertz Electronics for Sensing Applications
}

\author{
Michael Shur \\ Center for Integrated Electronics \\ Rensselaer Polytechnic Institute \\ Troy, NY 12180, USA \\ shurm@,rpi.edu
}

\begin{abstract}
Terahertz sensing is used for detection of hidden objects, explosives and biological and chemical hazardous agents, and for applications in radio astronomy, space research, biology and medicine. Emerging $\mathrm{THz}$ transistor sensing technology uses the rectification of electron density oscillations in short channel Si CMOS and FIN FETs and III-V and III-N High Electron Mobility Transistors (HEMTs). Such plasma wave electronics detectors might be tunable by applied bias voltage and can be modulated at very high frequencies. Using synchronized $\mathrm{THz}$ plasmonic transistor arrays is expected to improve performance of $\mathrm{THz}$ electronic detectors by orders of magnitude.
\end{abstract}

\section{INTRODUCTION}

Terahertz electronics could greatly expand applications of terahertz technology for sensing and imaging with big reductions in cost, system size, and power consumption compared to terahertz photonics systems. Plasmonic FET THz detectors have potential for outperforming other types of $\mathrm{THz}$ electronic detectors if the problems related to coupling and matching of $\mathrm{THz}$ radiation to the device, FET parasitics, interplay of contacts and ballistic transport in short channel structures, and high field properties of resonant and overdamped plasma waves will be understood and solved.

\section{TERAHERTZ SENSING}

\section{A. Applications}

Figure 1 lists frequency ranges for $\mathrm{THz}$ sensing applications.

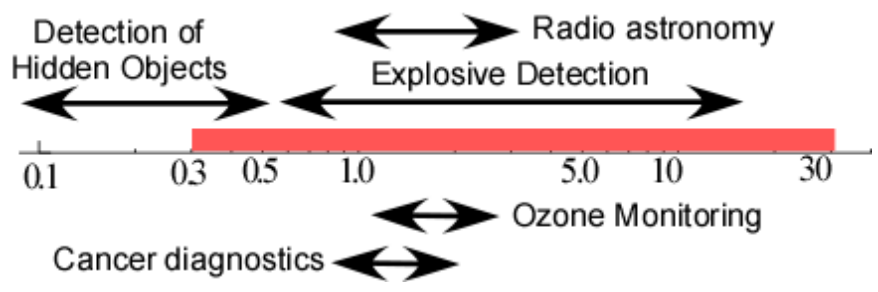

Fig. 1. THz sensing applications

This work is supported by the NSF under the auspices of I/UCRC "CONNECTION ONE" at RPI.

\section{B. THz detectors}

The bolometer (invented in 1878 by Samuel Pierpont Langley) was the first device capable of sensing $\mathrm{THz}$ radiation. Table 1 summarizes the characteristics of modern $\mathrm{THz}$ detectors.

TABLE I. TERAHERTZ DETECTORS

\begin{tabular}{|c|c|c|c|}
\hline THz detector & $\begin{array}{l}\text { Spectral } \\
\text { range } \\
(\mathrm{THz})\end{array}$ & $\begin{array}{c}\text { Operating } \\
\text { temperature } \\
(\mathrm{K})\end{array}$ & $\begin{array}{l}\text { Noise equivalent } \\
\text { Power }\left(\mathrm{W} / \mathrm{Hz}^{1 / 2}\right)\end{array}$ \\
\hline Si Bolometer $^{1}$ & $0.1-150$ & $0.3-4.2$ & $\begin{array}{c}2.4 \times 10^{-16}(@) \\
0.3 \mathrm{~K} \\
10^{-13}(@ 4.2 \mathrm{~K}\end{array}$ \\
\hline Pyroelectric $^{2}$ & $0.1-30$ & $240-350$ & $10^{-10}$ \\
\hline Golay Cell $^{3}$ & $0.3-15$ & 300 & $10^{-10}$ \\
\hline $\begin{array}{l}\text { Hot Electron } \\
\text { Bolometer }^{1}\end{array}$ & $0.1-70$ & 4.2 & $3 \times 10^{-13}-10^{-10}$ \\
\hline $\begin{array}{l}\text { Schottky } \\
\text { Diodes }{ }^{4}\end{array}$ & $0.1-3$ & 300 & $10^{-10}-10^{-12}$ \\
\hline $\mathrm{Si} \mathrm{CMOS}^{5}$ & $0.3^{*}$ & 300 & $10^{-11}$ \\
\hline $\begin{array}{c}\text { Si CMOS } \\
\text { heterodyne }^{6}\end{array}$ & $0.65^{*}$ & 300 & $8 \times 10^{-14}$ \\
\hline Si FINFET $^{7}$ & $0.2-2.5^{*}$ & 300 & $10^{-7}$ \\
\hline
\end{tabular}

$*$ it is expected to operate up to and above $10 \mathrm{THz}$ with re-design

In this paper, we will focus on $\mathrm{THz}$ electronics and, in particular, on emerging plasmonic detectors that have promise of using mainstream silicon CMOS based technology for THz applications.

\section{C. $\quad$ THz plasmonic detectors}

As was predicted in ${ }^{8}$, plasma waves (which are oscillations of the electron density) in the channels of short field effect transistors (FETs) might be unstable in a certain range of the FET currents and such short channel FETs could be used as sources and detectors of THz radiation. ${ }^{9,10}$

Dependent upon the incident $\mathrm{THz}$ frequency, semiconductor momentum relaxation time, radiation damping, and device dimension, the detection can be resonant (when the FET channel serves as a resonant cavity for the plasma waves) and non-resonant (when plasma waves are 
overdamped and the detection under bias is determined by the device nonlinear current voltage and capacitance-voltage characteristics. Resonant detection takes place when $\omega \tau>>1$ and $s \tau / L>>1$, where $\omega$ and $\tau$ are the angular frequency of the incident radiation and the plasmon lifetime time respectively, $s$ is the plasma wave velocity and $L$ is the FET channel length. The plasma wave velocity is $s=\left(q V_{g t} / m\right)^{1 / 2}$, where $q$ is the electronic charge, $m$ is the electron effective mass and $V_{g t}=V_{g s}-V_{t}$ is the gate voltage swing, and $V_{t}$ is the threshold voltage. Such resonant detectors are tunable by the gate bias. For $\omega \tau>>1$ but $s \tau / L<<1$, or for $\omega \tau<<1$, detection is non-resonant, (broadband).

So far, the room terahertz detection observed in silicon FETs has been non-resonant, (although at gate lengths sufficiently short and high enough frequency, room temperature resonant detection in silicon may be possible $\left.{ }^{11}\right)$. Resonant detection was observed in InGaAs HEMTs (see, for example, ${ }^{12-13}$ and AlGaN/GaN HEMTs. ${ }^{14}$ AlGaN/GaN plasmonic detectors recently demonstrated superior performance. ${ }^{15}$

Broadband $\mathrm{THz}$ detection using short channel FETs can be used in a wide temperature range, ${ }^{9,16}$. In this regime, a THz signal excites plasma waves decaying while propagating from channel boundaries. The characteristic decay length, ${ }^{17,18,19}$

$$
L_{o}=\sqrt{\frac{V_{g s}-V_{T}}{2 \pi \mu f}},
$$

determines the spatial resolution of such detectors when used for proximity sensors (see, for example, ${ }^{20}$ ) and could reach a nanometer resolution (see Fig. 2).

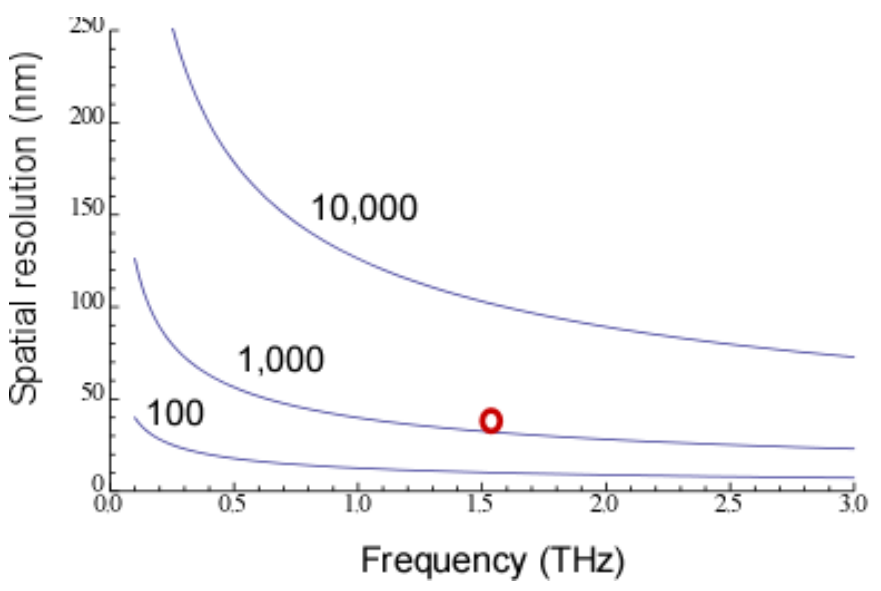

Fig. 2: Estimated spatial resolution of broadband plasmonic FET detector. The numbers next to the curves are the values of the low field mobility in $\mathrm{cm}^{2} / \mathrm{V}-\mathrm{s}$. The dot corresponds to the estimated resolution for $\mathrm{THz}$ detectors reported in ${ }^{7}$

The predicted and measured responsivities are quite high (see Figs. 3 and 4) and could be enhanced by biasing transistor close or even deep in the saturation regime due the increased non-linearity. ${ }^{21,18,19}$

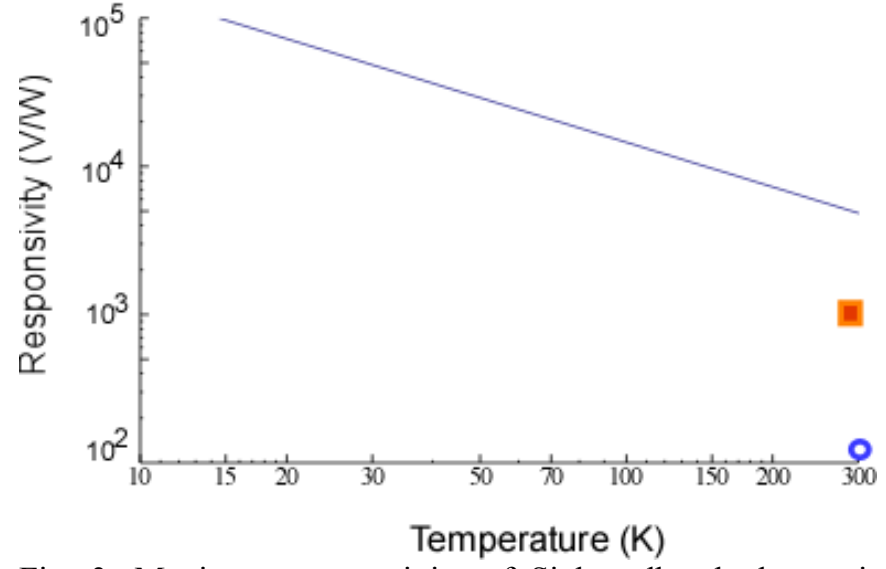

Fig. 3. Maximum responsivity of Si broadband plasmonic FET detectors (assuming perfect coupling) for zero drain current. Symbols correspond to the responsivities measured for Si FIN FETs at zero current and close to saturation. ${ }^{7}$

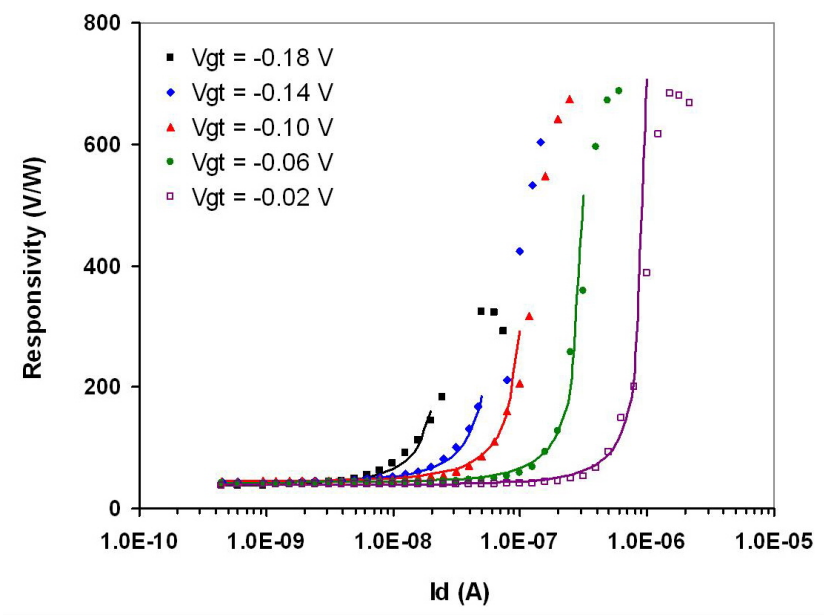

Fig. 4. $0.2 \mathrm{THz}$ responsivity of 20 fin device with $40 \mathrm{~nm}$ width and $100 \mathrm{~nm}$ gate length. Symbols are measured data; lines are modeled responsivity. ${ }^{7}$

Parasitics, such as the gate series resistance, play a crucial role and have to be minimized.

Grating gate structures could be used to capture the entire $\mathrm{THz}$ beam and enhance coupling, and pronounced plasmon resonances were observed at elevated temperatures up to $170 \mathrm{~K}$ in grated gate structures due to strong coupling between incident $\mathrm{THz}$ radiation and plasmons using the grating gate with relatively narrow slits ${ }^{22}$ (see Fig. 5) as predicted in ${ }^{23}$. The absorbance approached its theoretical maximum $A=0.5\left(1-\sqrt{R_{0}}\right)$, where $R_{0} \approx 0.25$ is the reflectivity from a bare sapphire substrate, at temperature 120 $\mathrm{K}$. The maximal absorption at the plasmon resonance is achieved when a half of the electron scattering rate becomes equal to the plasmon radiative decay rate. ${ }^{24}$

Preliminary results on the $\mathrm{THz}$ detection using multiple transistors show big enhancement in responsivity. ${ }^{25}$ Much greater improvements are expected from using plasmonic 
transistor arrays ${ }^{26,23}$ due to a much better coupling of $\mathrm{THz}$ radiation. The FET array acts as a broadband antenna, and coupling increases proportionally to the number squared of the FET in the array because the plasmon mode is spread over the entire FET array. ${ }^{24}$

Heterodyne plasma wave detection discussed in ${ }^{27}$ allows for a significant reduction in NEP. ${ }^{6,28}$
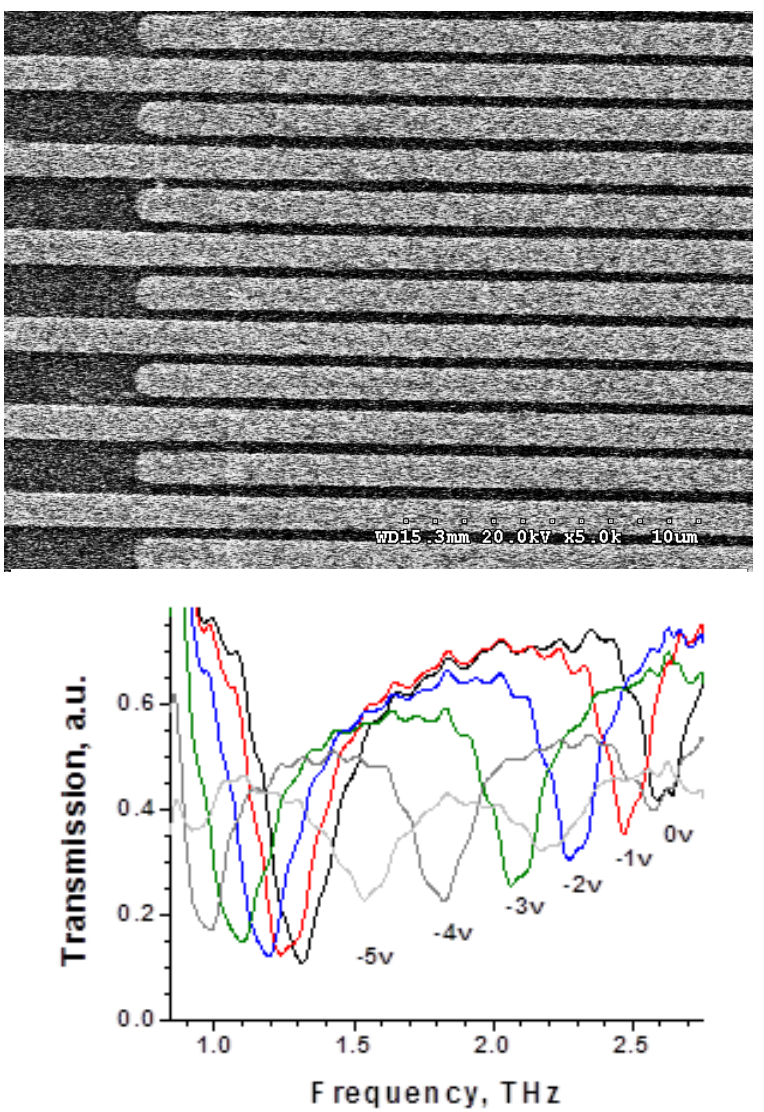

Figure 4. GaN-based 2D electron gas large area grating-gate HEMT structure on sapphire substrate. Electron concentration under the gates can be controlled by applying voltage on gate fingers. Terahertz beam had normal incidence and its polarization was perpendicular to the gate fingers. Experimental transmission spectra dependence on the gate voltage. Resolution is $0.1 \mathrm{THz}$. Temperature $=10 \mathrm{~K}{ }^{26}$

Images obtained by moving the transistor with respect to the $\mathrm{THz}$ beam reveal coupling patterns of the $\mathrm{THz}$ radiation (Fig. $5^{29}$ ). The resolution of such images could be as high as a few nanometers because the patterns are sensitive to the filed distribution at the gate edges determining the boundary conditions for the electron density oscillations. Impressive $\mathrm{THz}$ images were obtained in ${ }^{30,31}$.

Such high sensitivity to the electrical conditions at the gate edge makes plasmonic FETs to be extremely sensitive to biological agents as shown in ${ }^{20}$. Large changes in the $\mathrm{THz}$ response of plasmonic detectors loaded with solutions of globulin free bovine serum albumin (BSA), arginine and heparin were observed (compare Fig. 6 and 7). The same concentrations only very weakly affected the device transfer characteristics (see Fig. 7). Such change of the response characteristics can be used to identify and quantify biological and chemical substances makes this device suitable for using as a building block of a biochip with integrated processing capabilities.
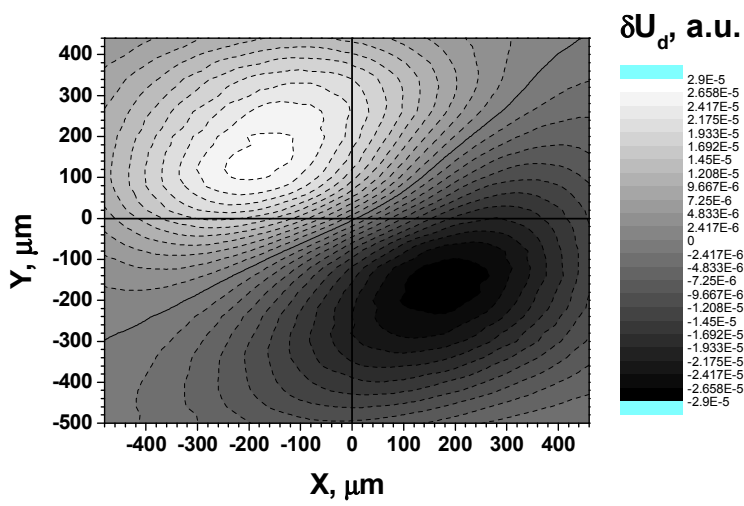

Fig. 5. THz image of a transistor, recorded as the dependence of the transistor response with its displacement in the focal plane in $5 \mu \mathrm{m}$ steps. $f=1.63 \mathrm{THz}$; the polarization of $\mathrm{THz}$ radiation is perpendicular to the gate (vertical axis). $\left(\right.$ From $^{29}$ )

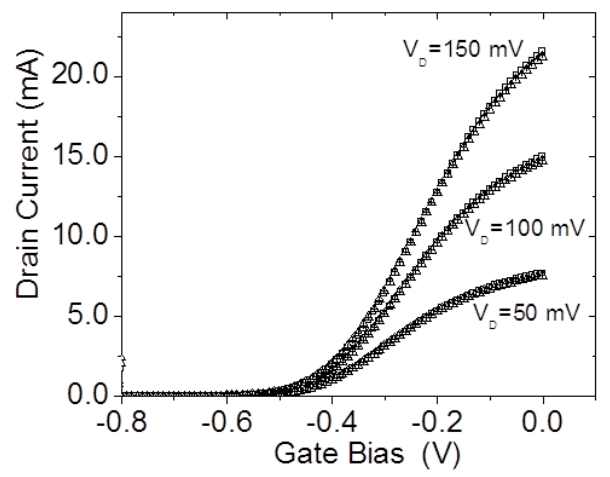

Fig. 6. Transfer characteristics of $\mathrm{AlGaAs} / \mathrm{GaAs}$ HEMT plasmonic $\mathrm{THz}$ detectors before and after covered with biological specimens (marked by different symbols) (from ${ }^{20}$ ).

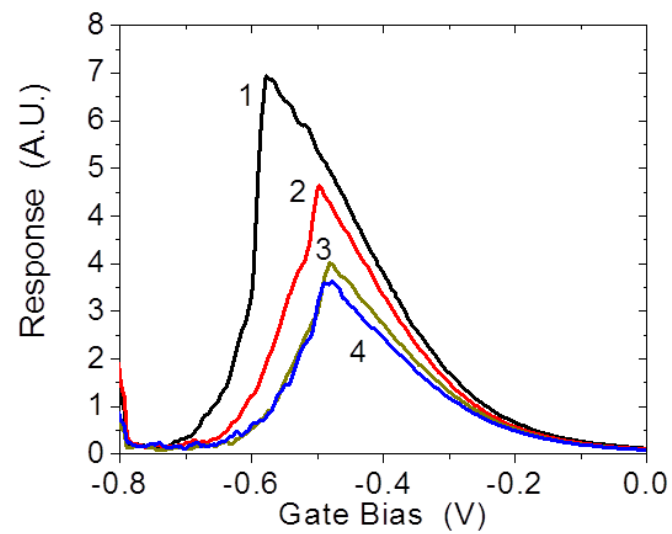

Fig. 7. $\mathrm{THz}$ response of plasmonic detector after covering with heparin solutions with different concentrations: 1)before biological specimen 2) $1 \mathrm{mg} / \mathrm{ml} \mathrm{3)} 2 \mathrm{mg} / \mathrm{ml} \mathrm{4}) 3 \mathrm{mg} / \mathrm{ml}\left(\right.$ from $^{20}$ ) 


\section{ACKNOWLEDGMENT}

I am grateful to my colleagues Drs. M. Dyakonov, W. Knap, A. Muraviev, W. Stillman, D. Veksler, S. Rumyantsev, A. Dmitriev, V. Kachorovskii, F. Teppe, V. Ryzhii, V. Popov, T. Otsuji, and N. Pala, for their pioneering contributions to plasma wave $\mathrm{THz}$ electronics.

\section{REFERENCES}

[1] Inside Product Co. http://www.insight-product.com/detect3.htm

[2] Spectrum Detector, Inc., http://www.spectrumdetector.com/pdf/datasheets/THZ.pdf (2007)

[3] QMC_Instruments, http://www.terahertz.co.uk/ (2010)

[4] Virginia_Diodes_Inc,http://virginiadiodes.com/WR2.2ZBD.htm, 2007

[5] F. Schuster, D. Coquillat, H. Videlier, M. Sakowicz, F. Teppe, L. Dussopt, B. Giffard, T. Skotnicki, and W. Knap, Broadband terahertz imaging with highly sensitive silicon CMOS detectors. Optics Express. 2011 Apr 11;19(8):7827-32 (2011)

[6] D. Glaab, S. Boppel, A. Lisauskas, U. Pfeiffer, E. Öjefors, and H. G. Roskos, Terahertz heterodyne detection with silicon field-effect transistors, Appl. Phys. Lett. 96, 042106 (2010)

[7] W. Stillman, C. Donais, S. Rumyantsev, M. Shur, D. Veksler, C. Hobbs, C. Smith, G. Bersuker, W. Taylor and R. Jammy, Silicon FIN FETs as detectors of terahertz and sub-terahertz radiation, International Journal of High Speed Electronics and Systems, vol. 20, No. 1, pp. 2742 March (2011)

[8] M. Dyakonov and M. Shur, "Shallow water analogy for a ballistic field effect transistor: New mechanism of plasma wave generation by dc current," Physical Review Letters, vol. 71, pp. 2465-2468 (1993)

[9] M. I. Dyakonov and M. S. Shur, "Plasma wave electronics: Novel terahertz devices using two dimensional electron fluid," IEEE Transactions on Electron Devices, vol. 43, pp. 1640-1645 (1996)

[10] M. Dyakonov and M. Shur, "Detection, mixing, and frequency multiplication of terahertz radiation by two-dimensional electronic fluid," IEEE Transactions on Electron Devices, vol. 43, pp. 380-387 (1996)

[11] Y. Deng and M. S. Shur, Electron Mobility and Terahertz Detection using Silicon MOSFETs, Solid-State Electronics, Vol. 47, Issue 9 , pp. 1559-1563, September (2003)

[12] J.-Q. Lu, M. S. Shur, J. L. Hesler, L. Sun, and R. Weikle II, A Resonant Terahertz Detector Utilizing a High Electron Mobility Transistor, IEDM Technical Digest, 879-882 (1998)

[13] W. Knap, A. El Fatimy, J. Torres, F. Teppe, M. Orlov, and V. Gavrilenko, Plasma wave resonant detection of terahertz radiations by nanometric transistors, Low Temp. Phys. 33, 291-294 (2007)

[14] El Fatimy, S. Boubanga Tombet, F. Teppe, W. Knap, D. B. Veksler, S. Rumyantsev, M. S. Shur, N. Pala, R. Gaska, Q. Fareed, X. Hu, D. Seliuta, G. Valusis, C. Gaquiere, D. Theron, and A. Cappy, Terahertz detection by GaN/AlGaN transistors, Electron. Lett. 42, 1342 (2006)

[15] T. Tanigawa, T. Onishi, S. Takigawa, and T. Otsuji, Enhanced Responsivity in a Novel AlGaN/GaN Plasmon-Resonant Terahertz Detector Using Gate-Dipole Antenna with Parasitic Elements, presented at the DRC 2010, paper no. IV.A-9, at the University of Notre Dame, South Bend, IN, June 21 to 23 (2010)
[16] M. I. Dyakonov and M. S. Shur, Detection, Mixing, and Frequency Multiplication of Terahertz Radiation by Two Dimensional Electronic Fluid, IEEE Transactions on Electron Devices, Vol. 43, No. 3, pp. 380387, March (1996)

[17] W. Knap, Y. Deng, S. Rumyantsev, and M. S. Shur, Field effect transistor as heterodyne terahertz detector, Appl. Phys. Lett, 81, 46374639 (2002)

[18] D. Veksler, F. Teppe, A. P. Dmitriev, V. Y. Kachorovskii, W. Knap, and M.S. Shur, Detection of terahertz radiation in gated twodimensional structures governed by dc current, Phys. Rev. B 73, 125328 (2006)

[19] T.A. Elkhatib, V.Yu. Kachorovskii, W.J. Stillman, S. Rumyantsev, X.C. Zhang, and M. S. Shur, THz Response of Field-Effect Transistors in the Saturation Regime, Appl. Phys. Lett., 98, 243505-243507 (2011)

[20] N. Pala and M. Shur, Plasmonic $\mathrm{THz}$ detectors for biodetection, Electronics Letters, 20 November -- Vol. 44, Issue 24, p. 1391-1393 (2008)

[21] J.-Q. Lu and M.S. Shur, Terahertz Detection by High Electron Mobility Transistor: Effect of Drain Bias, Appl. Phys. Lett, Vol. 78, 2587-2589 (2001)

[22] A.V. Muravjov, D.B. Veksler, V.V. Popov, O. Polischuk, X. Hu, R. Gaska, N. Pala, H. Saxena, R.E. Peale, M.S. Shur, Temperature dependence of plasmonic terahertz absorption in grating-gate $\mathrm{GaN}$ HEMT structures, Appl. Physics Lett, 96, 042105 (2010)

[23] V. V. Popov, M. S. Shur, G. M. Tsymbalov, and D. V. Fateev, Higherorder plasmon resonances in GaN-based field-effect transistor arrays, International Journal of High Speed Electronics and Systems, vol. 17, No. 3 pp. 557-566, September (2007)

[24] V.V. Popov, G.M. Tsymbalov, D.V. Fateev, M.S. Shur, Cooperative absorption of terahertz radiation by plasmon modes in an array of fieldeffect transistors with two-dimensional electron channel, Appl. Phys. Lett. 89, 23504 (2006)

[25] T. A. Elkhatib, V. Yu. Kachorovskii, W. J. Stillman, D. B. Veksler, K. N. Salama, X.-C. Zhang, and M. S. Shur, Enhanced Plasma Waves Detection of Terahertz Radiation using Multiple High-ElectronMobility Transistors Connected in Series, IEEE Trans. Microwave Theory and Techniques, vol. 57, issue 2, pp. 331-339 (2010)

[26] M. I. Dyakonov and M. S. Shur, Two Dimensional Electronic Flute, Appl. Phys. Lett, Vol. 67 (8), August 21, pp. 1137-1139, (1995)

[27] B. Gershgorin, V.Yu. Kachorovskii, Y. V. Lvov, and M. S. Shur, Field effect transistor as heterodyne terahertz detector, Electronics Letters, vol. 44, No. 17 (2008)

[28] U. Pfeiffer, E. Öjefors, A. Lisauskas, D. Glaab, and H. G. Roskos, A CMOS focal-plane array for heterodyne terahertz imaging, Proc. IEEE RFIC Symposium 2009, 433-436 (2009)

[29] D.B. Veksler, A.V. Muravjov, V.Yu. Kachorovskii, T.A. Elkhatib, K.N. Salama, X.-C. Zhang and M.S. Shur, Imaging of field-effect transistors by focused terahertz radiation, Solid-State Electronics, vol. 53, 571 (2009)

[30] F. Schuster, W. Knap, and V. Nguyen,Terahetz Imaging achieved with low-cos CMOS detectors, Laser Focus World, pp. 37-41, July (2011)

[31] E. Ojefors, U.R. Pfeiffer, A. Lisauskas, and H.G. Roskos, A $0.65 \mathrm{THz}$ Focal-Plane Array in a Quarter-Micron CMOS Process Technology, IEEE Journal of Solid-State Circuits, vol: 44 Issue:7, pp. 1968 - 1976, July (2009) 